Article

\title{
Opportunities for Industry 4.0 to Support Remanufacturing
}

\author{
Shanshan Yang, Aravind Raghavendra M. R. *, Jacek Kaminski and Helene Pepin
}

Advanced Remanufacturing and Technology Centre (ARTC), Agency for Science, Technology and Research (A*STAR), 3 CleanTech Loop, \#01/01, CleanTech Two, Singapore 637143, Singapore; yangs@artc.a-star.edu.sg (S.Y.); kaminskijk@artc.a-star.edu.sg (J.K.); helene-pepin@artc.a-star.edu.sg (H.P.)

* Correspondence: aravindr@artc.a-star.edu.sg; Tel.: +(65)-6908-7911

Received: 28 June 2018; Accepted: 13 July 2018; Published: 19 July 2018

\begin{abstract}
Remanufacturing is the process of bringing end-of-life products back to good-as-new. It plays a critical role in decoupling economic growth from growth in resource use, and in accelerating the circular economy. However, the uptake of remanufacturing activities faces obstacles. This paper reviews the challenges encountered by the remanufacturing sector and discusses how the Industry 4.0 revolution could help to effectively address these issues and unlock the potential of remanufacturing. Two case studies are included in this paper to exemplify how technology enablers from Industry 4.0 can increase efficiency, reliability, and digitization of the remanufacturing process.
\end{abstract}

Keywords: remanufacturing; sustainable manufacturing; Industry 4.0; smart factory; intelligent machining

\section{Introduction}

The growth and stability of the traditional "take-make-consume-dispose" linear model has been heavily dependent on the availability of resources. However, the linear model is now challenged by an unprecedented rise in demand for the finite supply of resources, as it is expected that by 2030 there will be three billion more middle-class consumers worldwide [1]. With this in mind, the "circular economy" model is drawing global attention as an approach to decouple economic growth from resource constraints. The underlying principle of the "circular economy" is to make products and materials restorative and regenerative by design, and to maintain them at their highest utility and value [2].

Circulation of the technical product life cycle is enabled through several end-of-life (EOL) channels, including recycling, remanufacturing, reusing, refurbishing, etc. Among these EOL strategies, remanufacturing shows noticeable advantages due to its effectiveness in preserving the added value of products and assuring their quality. Remanufacturing is the process of bringing EOL products back to good-as-new status through disassembly, cleaning, inspection/sorting, restoring, and reassembly. It provides social, environmental, and economic benefits by offering to consumers end products with assured quality at a competitive price, while protecting the intellectual property and brand image of original equipment manufacturers (OEMs). It also creates and opens up new business and job opportunities in the after-sales service market. Another main advantage of remanufacturing lies in protection of the environment by reducing the usage of raw materials, carbon footprint, and number of components being scrapped. However, the uptake of remanufacturing faces several obstacles, which need to be resolved properly through collaboration among multiple players across the business, government, investors, society, and research communities [3]. Fortunately, the advent of Industry 4.0 has provided immense opportunities for unlocking the potential for remanufacturing by reducing the cost of transformation into a higher level of connectivity and efficiency. 
Industry 4.0, as the name suggests, refers to the fourth stage of industrialization, aiming for a high level of automation in the manufacturing industry through the adoption of ubiquitous information and communication technologies (ICTs). The boundaries between the virtual environment and real world get increasingly blurred, which is called "cyber-physical production systems (CPPSs)". In simple terms, in CPPSs, electronic and mechanical components are linked through sensors in a network, which provides a smart platform for data flow and data analytics. An early form of this technology is the implementation of radio frequency identification (RFID) sensors, which have been in wide use since the year 1999.

In this paper, the opportunities that Industry 4.0 bring to the remanufacturing industry are discussed and presented from the perspectives of "Smart Life Cycle Data", "Smart Factory", and "Smart Services". Case studies are presented to exemplify the use of ICT to increase the efficiency and accuracy of the remanufacturing process.

\section{Literature Review}

Remanufacturing activities span a number of industries. Remanufacturing has experienced a higher chance for success in sectors in which:

- Products are durable and usually contain high-value materials;

- The technology cycle is stable and longer than the useful life cycle;

- Restoration technologies are available;

- Products have the potential to be leased or delivered as a service rather than as hardware.

The above-mentioned factors are also the reasons why more than $60 \%$ of remanufacturing activities, in terms of production value, are concentrated in the aerospace, automotive, and heavy-duty off-road vehicle sectors. Examples of products or components being remanufactured include aerospace engines, alternators for automotive, gears for heavy-duty vehicles, etc. More recently, driven by noticeable economic profits and ever more stringent environmental regulations, remanufacturing has also been carried out by other industries, such as IT products, machinery, tires, and furniture.

Bottlenecks to remanufacturing vary by geographic location, industry sectors size of the individual remanufacturer, and nature of business. Nevertheless, there are still some common challenges, which are identified as follows:

- Lack of standards and legislation

The lack of a commonly accepted definition and standards for remanufactured products in various sectors has been identified as the most prevalent barrier to remanufacturing. This has resulted in not only the loss of consumer trust in remanufactured products, but also restrictions on importing or exporting remanufactured products in certain countries [4].

- $\quad$ Lack of life cycle design awareness

Many barriers encountered during the remanufacturing process could be eliminated if proper design features were included in the early stage of product design. For example, avoiding the use of permanent joints reduces the complexity of disassembly processes, and standardizing part designs simplifies the inspection and sorting process. Currently, most design for remanufacturing (DfRem) tools or concepts remain within the realm of academic research. There is still a lack of an effective DfRem tool commonly accepted and adopted by industry, which hinders the closing of the product life cycle through remanufacturing [5].

- Lack of sufficient market demand and core supply

"Cores" refer to the used products which are re-collected for remanufacturing. A lack of understanding and negative perception of remanufacturing have limited market demand for remanufactured products. When customers are not convinced of the quality of remanufactured 
products, they expect remanufactured products to be sold at a lower price, which consequently reduces the profit margin and discourages growth of the remanufacturing industry. Furthermore, due to the current linear movement of product from the point of manufacture to customers and, ultimately, to landfills, incineration, or material extraction, gaining access to EOL products and diverting qualified ones to a remanufacturing facility also pose challenges to the growth of remanufacturing. There is a need to change to circular or service-oriented models that can truly reverse the linear trend and assure a supply of cores [4].

- Skill/technology challenges and limited information sharing

Remanufacturing is a highly labor-intensive industry in comparison with traditional manufacturing [6]. Many of the decisions made during the remanufacturing process are still dependent on ad hoc engineering experience and thus require technically skilled engineers or technicians. Meanwhile, there is still a need for the development of suitable nondestructive testing (NDT) methods for repaired components or parts, which makes the qualification of remanufactured products challenging. In addition to this, the lack of original design specifications and information on the usage and repair history of the returned products further complicate the assessment of the viability of core remanufacturing.

Even though progress towards the uptake of remanufacturing is still hampered by a few challenges, Industry 4.0 reveals some powerful emerging trends that have the potential to address these bottlenecks. While Industry 3.0 focused on the automation of a single machine and process, Industry 4.0 pushes towards end-to-end digitalization of all physical assets and the entire supply chain [7]. The rise of Industry 4.0 is leading to the drastic and rapid growth of data volume (Big Data), driven by:

- The availability of computing power and connectivity,

- The advancement of analytic capability (e.g., artificial intelligence),

- The introduction of new patterns of human and machine interaction (e.g., augmented reality systems), and

- The advent of technologies that ease the transformation of digital data into physical objects (e.g., additive manufacturing and rapid prototyping).

When it comes to digitalization, Industry 4.0 possesses four main characteristics:

- The vertical networking of small production systems, such as smart factories and smart products;

- The horizontal integration of global value creation networks, such as new business and cooperation models;

- Through-engineering across the product design, product use, and EOL stages;

- Exponential acceleration of technologies.

The fundamental idea of Industry 4.0 is to increase availability and integrated use of relevant data by connecting all products, resources, and companies involved in the value chain and, ultimately, to generate additional value from available data and to maximize customer benefit [8]. According to a recent global industry survey [7], Industry 4.0 is no longer considered a "future trend" and for many companies it is already the core of their strategy and research agenda. Around $40 \%$ of companies surveyed reported that their vertical and horizontal value chains and through-engineering model are already benefiting from an advanced level of digitization and integration; this number is expected to increase to $75 \%$ within 5 years. Meanwhile, survey results suggested that global industrial product companies would invest USD907 billion per year through to 2020 for digital transformation. It is believed that the digitization of horizontal and vertical value chains, as well as the product life cycle, will eventually revolutionize the product, manufacturing/remanufacturing industry, and corresponding business models. 


\section{Smart Remanufacturing in the Digital Age}

To pave the way towards a wider uptake of remanufacturing, the challenges discussed in the previous section need to be addressed and properly resolved by OEM and independent remanufacturers. Fortunately, the advent of Industry 4.0 has presented immense opportunities to unlock the potential of remanufacturing. In this section, the opportunities that Industry 4.0 bring to remanufacturing are discussed based on the three aspects previously mentioned, namely, Smart Life Cycle Data for Design for Remanufacturing and EOL Management, Smart Factory for cost-effective and green remanufacturing operations, and Smart Services for a successful remanufacturing business model. Figure 1 describes the three application areas and technical enablers from Industry 4.0. The technology enablers are presented in the outer rim of the circle, and include smart sensors, cloud computing, robotics, machine-to-machine communication (M2M), additive manufacturing, and others.

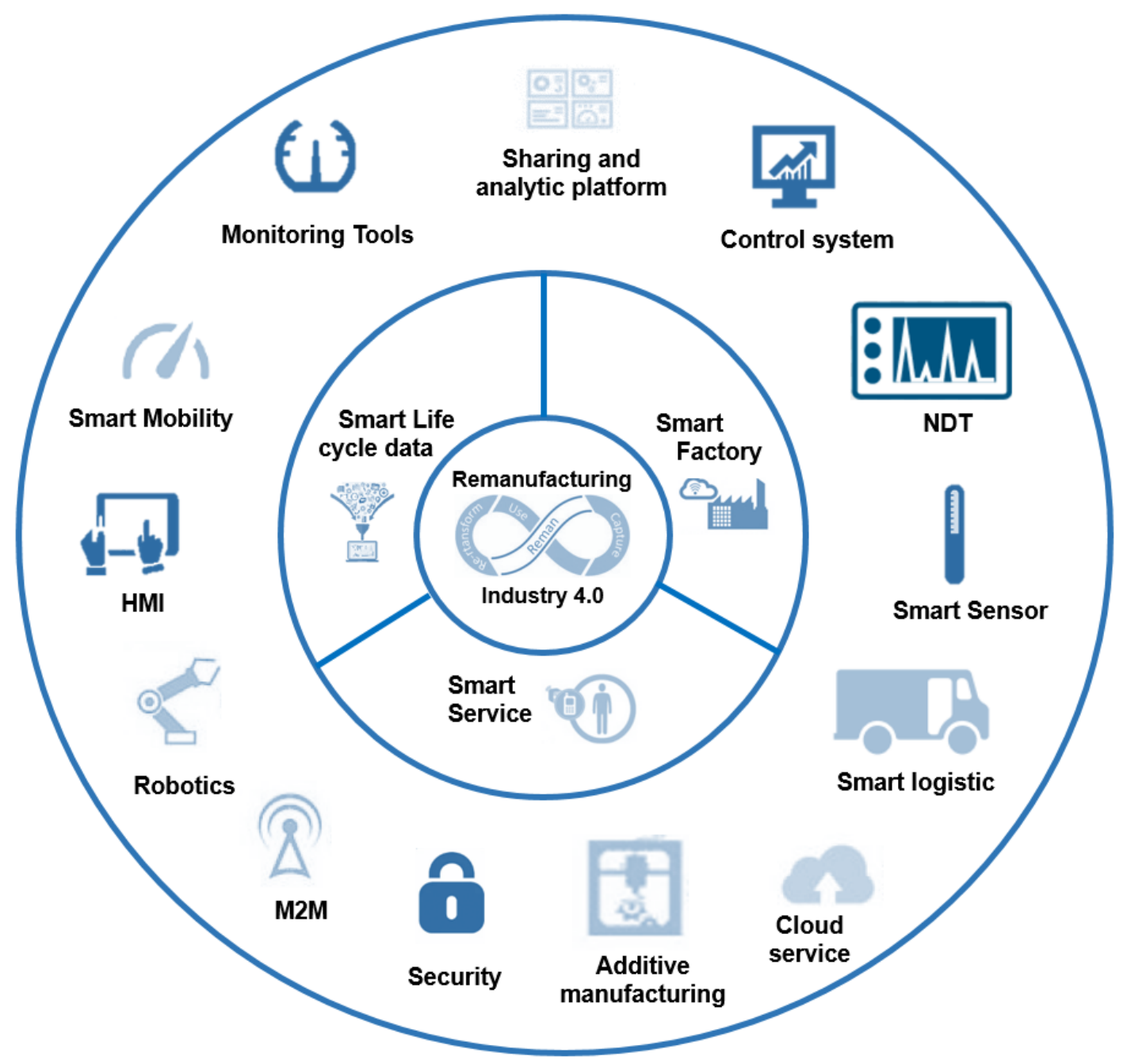

Figure 1. Opportunities from Industry 4.0 for remanufacturing, and its key enablers.

\subsection{Smart Life Cycle Data}

From the initial product design and development stage, and all the way until the EOL stage, various product information is generated and captured. Ideally, this information should be shared across the product life-cycle stakeholders to support product life-cycle management [5]. However, the flow of product information remains essentially unestablished due to ineffective data extraction, loss of information during product transfer between stakeholders, undeveloped platforms 
to support information sharing, and other policy restrictions. Ineffectiveness of data circulation has reduced the efficiency of product life-cycle management and the quality of service provided. For example, incomplete information on returned cores remains a big challenge for the majority of remanufacturers [5]. In order to restore these products back to "good as new" quality, remanufacturers have to recreate product knowledge which existed at the product design stage. In this regard, the digital transformation of Industry 4.0 has shed some light on addressing this concern by improving data transferability and building the knowledge/data-sharing platform. This could be enabled through sensors, embedded systems, and connected devices ("Internet of Things"), as well as a comprehensive data management platform. For example, when information regarding computer-aided design (CAD), bill of materials, parts information, manufacturing and assembly instructions, data from product use stage, and repair history information are stored in a central system and are easily accessible by remanufacturers, the repair decisions during the remanufacturing process could be made easily and operations could be carried out in a more efficient manner. Furthermore, when information, such as product failure modes and rates, replacement frequency, cleaning efficiency, disassembly challenges, and upgrading challenges, is extracted effectively from the remanufacturing stage and fed back to product designers, many of the barriers occurring during the remanufacturing process could be avoided in the next generation of products by incorporating proper design features [9-12]. This is strategically important and a substantial cost-saving measure, as more than $70 \%$ of product costs are determined at the product development stage [13].

\subsection{Smart Factory}

Due to the uncertainty involved in the number and quality of cores returned, remanufacturing operations need to incorporate a high degree of flexibility to react quickly and appropriately to various product reconditioning requirements. Smart factories, which enable high flexibility and small batch size production, seamlessly address this complexity issue associated with remanufacturing operations. "Smart factories" are essentially at the core of Industry 4.0. "Smartness" is achieved using electronic hardware/software, as well as networking of production resources. Compared with traditional manufacturing, more ancillary hardware and software, like RFID tags, barcodes, laser markers, sensors, as well as communication infrastructure, will be embedded into the factory to enable machines to collaborate with each other using intelligent analytics. In the future, in a smart remanufacturing environment, machines could obtain incoming core information through scanning a barcode attached to the core, adapt the remanufacturing operations through self-optimization and smart fixturing capabilities, update the process-related information to a database via wireless transfer, and store remanufacturing knowledge gained from experience. This could enable a substantial reduction of the labor force and lessen the dependency on high-skilled operators. In the meanwhile, with various types of sensors embedded into equipment and cells, data from the manufacturing process could be retrieved and sent for real-time analysis. This would support the early detection of machines or cell failures and allow preventative strategies to be implemented to avoid unplanned maintenance and catastrophic failures. Information associated with the product manufacturing data could also been recorded and stored as part of the product life-cycle data. In addition, energy-efficient remanufacturing processes could also be achieved through collecting real-time energy consumption data and implementing an energy management system accordingly. Further innovative technologies, such as additive manufacturing, 3D scanning, automated guided vehicles, inspection drones, hybrid manufacturing/process [14,15], and augmented reality tools, will continuously drive down the cost of remanufacturing operations while also delivering substantial improvements in the quality of the repaired product. Taking aerospace remanufacturing as an illustration, worn airfoils can sometimes be repaired using the laser metal deposition technique, an additive process where metal powder is melted by a computer numerical control (CNC) laser on a robot arm to form a direct fusion-bonded deposit on the blades. This technology offers the precision and low heat input necessary to successfully achieve such restoration, compared to more conventional fusion welding 
processes. In addition, sustainability needs to be an important factor to be considered while adopting these advanced technologies. The life-cycle assessment method could be conducted to gauge the environmental impact of these new technologies to make sure the advancement is not only technically achieved but is also environmentally friendly [16].

\subsection{Smart Services}

One of the challenges that remanufacturers face, as explained earlier, is the control of the timing, quality, and quantity of cores returned. In this regard, the product service system has provided opportunities to cope with the complexity of core return for remanufacturing [17]. In this emerging and disruptive business model, ownership of the product is usually retained by the OEMs or retailers and only the service or usage is offered to customers (e.g., selling "flying hours of the engine" instead of selling "engines"). Hence, it creates a mandate for manufacturers or retailers to monitor their product's performance during its runtime and to forecast remanufacturing operations on the cores returned based on the predicted remaining life of the product. On the other hand, from the consumers' perspective, as they pay for the service rather than the ownership of the product, market acceptance for remanufactured goods will likely be increased, leading to a successful remanufacturing model. Real-time monitoring of product in-use and data analysis via embedded sensor networks and cloud-based computation could enable predictive maintenance by the early detection of problems. Increased connectivity among products, customers, and manufacturers, promoted by Industry 4.0, presents immense opportunities for boosting the product service model. Take, for example, machines that are leased to the power plant sector. Power plants rely heavily on the continuous availability of their machinery. To increase the reliability of machines, smart sensors are embedded into the machines to monitor critical factors, such as temperature, pressure, switches, energy consumption, and vibration in real time [18]. Sensor data are collected and logged into a central server through a network for prediction of potential wear and estimation of components' useful life, and for scheduling maintenance or remanufacturing of components in a timely manner.

\section{Case Illustrations}

As mentioned previously, traditional remanufacturing is hindered by low process efficiency and data silos among various product life-cycle stages. In this section, we present two case studies to demonstrate how Industry 4.0 helps traditional remanufacturing achieve better connectivity between machines through seamless data flow and real-time data capturing for condition monitoring, respectively.

\subsection{Smart Repair Cell}

Currently, most remanufacturing tasks are highly labor intensive, as returned cores vary greatly in terms of their form and condition. Even though human intelligence is capable of adapting to such variations and performing adaptive manual repair tasks, dealing with variability in the part profile and form accuracy of each core still remains challenging for the remanufacturing process. This is particularly a concern for aerospace part repair, as the sector has stringent requirements for compliance to the original part specifications.

To achieve consistency and high flexibility in remanufacturing operations, a smart remanufacturing cell, with special focus on repair operations, has been simulated at the Advanced Remanufacturing and Technology Centre (ARTC). The smart cell, as graphically described in Figure 2, comprises the following layers:

- Shared database - in this layer, all virtual data pertaining to every individual repaired component are stored and organized based on the design and existing repair information. By organizing the repair data, backtracking the core's repair process history and failure information is a well-established process. 
- Software platform - this layer defines all the software tasks related to the data flow and machine-to-machine communication channels. With a common software platform, high-speed computations are carried out both in real time and offline, helping seamless data transfer between machines without any loss in data quality.

- Physical process - this layer of the smart cell defines the actual repair tasks to be carried out on the core. Since the process requires physical transfer of the component and its storage container across the actual repair processes, it relies heavily on factory automation to conform to Industry 4.0 standards.

In standard manufacturing protocols, design of the component using computer-aided design (CAD) precedes part manufacturing. Yet, in the remanufacturing process, the type and level of damage to the core will affect repair decisions. Some of the damage modes include, but are not limited to: cracking, wear, physical distortion, high-temperature damage, surface corrosion, etc. Upon entering the cell, cores will be registered in the shared database using identification markers during a process termed "Part Registration". This process provides the system with core information, such as original part number, serial number, raw material identification, bill of materials (if it is an assembly), OEM CAD design information, and repair history.

Once cores are registered, a single/series of repair processes will be planned for each core in a sequence suitable for its identification and condition, based on established standard OEM repair protocols or other approved methods. The repair sequence will involve the utilization of several machines and manufacturing processes, such as brazing, thermal/cold spray, and laser cladding to regenerate the damaged area of the core. Process-related information will be updated in the database via wireless transfer and will constitute the digital "life-story" of the parts. This registration process is crucial in product life-cycle management (PLM) to improve the product design in the future and to ensure an appropriate decision-making process at the next remanufacturing cycle.

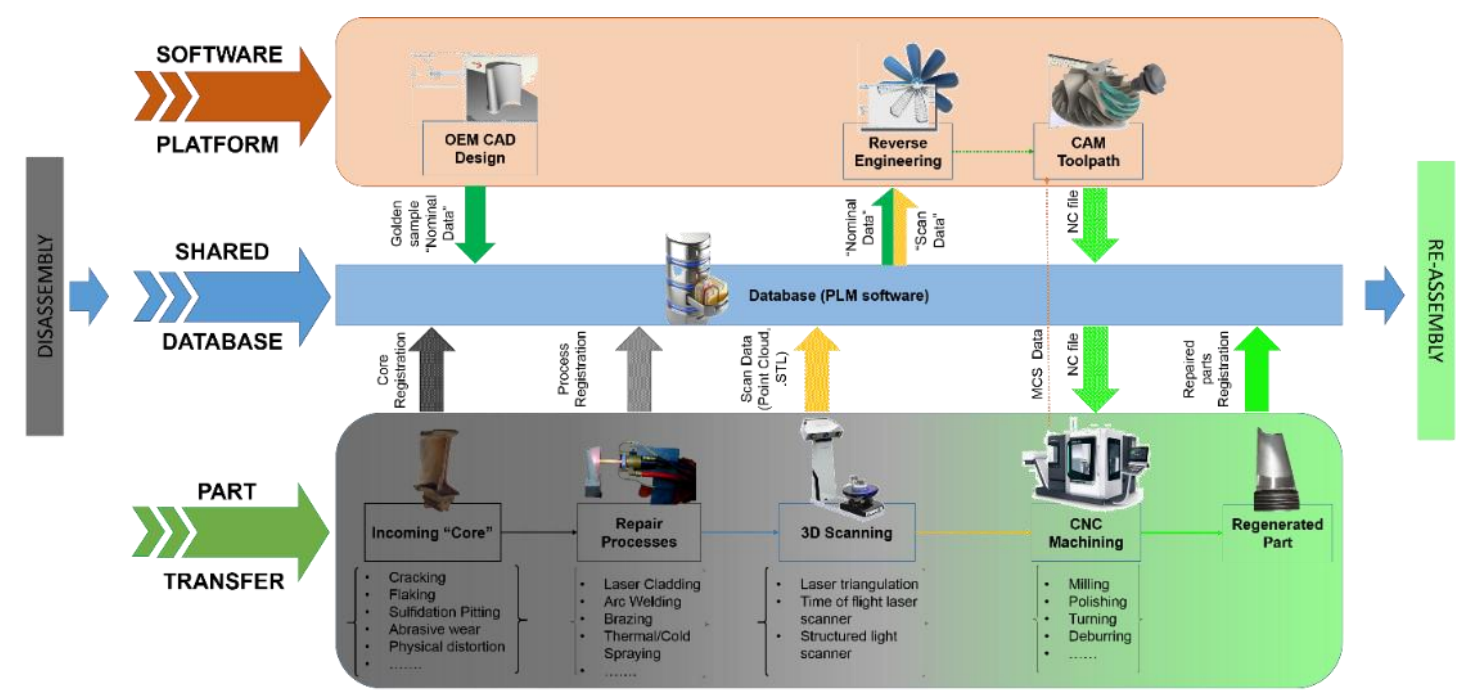

Figure 2. Smart remanufacturing cell-process flow.

As most of the repair processes result in addition of material to the parts, further machining processes need to be carried out to bring the repaired component back to its original dimensions. This is a critical stage of the repair process, as it will determine the accuracy and tolerances of the final repaired part. To generate the optimum tool path, automated adaptive machining is adopted. Firstly, the as-received physical dimensions of the core need to be defined in 3D digital format. This could be realized using various three-dimensional scanning methods, such as laser triangulation, structured light, probing methods, etc., to create the digital twin of the core. The scanned 
output is stored in the shared database, usually in point-cloud form. Secondly, the scanned data is compared with the original digital design of the part (computer-aided model-CAD), also called golden sample/nominal data, to obtain the geometry difference, using reverse engineering software. Thirdly, on-machine probing or a scanning method is carried out to measure the information on the machine coordinate system (MCS) and to align the repaired part with respect to the machine datum. Lastly, via the developed machine-to-machine communication software platform, geometry differences and the MCS are fed into computer-aided manufacturing (CAM) software for the adaptive tool-path generation. The tool path generated based on this aligned datum is stored in the shared database layer as a numerical control (NC) file. Following the additive repair processes, when a part is ready for final machining, the NC file is retrieved from the database to be used for machining. Various machining processes, such as 5-axis milling, turning, grinding, abrasive polishing, etc., are carried out to achieve the final dimensions on the repaired component. Machining-related information is also stored in the database as part of the product's digital "life-story". The real-time monitoring of the machining data plays a key role in defining the surface integrity of the machined parts. In the subsequent case study, the effect of sensor integration on machine tools and its impact on Industry 4.0 is discussed in detail.

In addition, thanks to the advancement of machine tool technology, both additive and subtractive processes can be performed with one single machine. Hybrid machines-combining both laser metal deposition blown powder and 5-axis milling in one machine-are now commercially available for use in production. This provides the benefit of single-machine and single-work setup, hence improving the volumetric accuracy of the final repaired component. In addition, the cost of multiple machines, corresponding part transfer automation, and the space requirement are reduced substantially.

Smart repair cells are designed to be an effective way of repairing each component adaptively with utmost accuracy and tolerance due to seamless information flow across the processes. Currently, a smart repair cell concept is simulated at ARTC using various scenarios such as number of machine tools, robotic automation vs manual part transfer, and volume of cores. Based on the simulated results, the best operational efficiency is calculated to select the right hardware combinations. Know-how in reverse engineering, adaptive toolpath development, and individual repair processes have already been embedded into the smart repair cell. Ongoing work at ARTC is focused on building up the database based on number of cores, real-time machine tool monitoring, and the final remanufactured part conformance data, which will eventually help machine learning for automatic repair decision making. It should be noted that the above-mentioned remanufacturing cell focuses mainly on the adaptive repair process. Disassembly, cleaning, re-assembly, and, most critically, the NDT process for analyzing issues related to adhesion of the repaired material, change of material structure, porosity, inclusions, etc. are not addressed in this paper.

\subsection{Sensorized Machines for Intelligent Machining}

Machine tools could become increasingly smart by perceiving their own states and the state of the surrounding environment, which is considered as "intelligent machining". Key enablers for this capability include smart sensors embedded into machine systems, a data acquisition system which can transmit the processing data quickly without data loss, and also the intelligent central system which receives and analyzes the data. Such an intelligent machining system is of great importance for evaluating the system's health and to enable prediction of a possible breakdown or malfunction before it happens. Currently, advanced condition-based maintenance is triggered when an asset reaches a predefined unacceptable level detected by the sensoring system. These thresholds usually originate from experts' experiences (machine operators) or manufacturers' recommendations. However, when multiple complicating factors coexist, false alarms might be triggered. To resolve such complications, sensors and advanced computer systems will be used in tandem during intelligent machining to monitor the system health. Both historical and real-time sensor data will be used in the evaluation of the machine condition via artificial intelligence techniques to substantially increase the 
prediction accuracy and the quality of the produced part. This work is now being carried out by a research group in ARTC.

A large number of sensing devices, including vibration sensors, current sensors, temperature sensors, and acoustic emission sensors, have been installed into various critical subsystems of a computer numerical control (CNC) machine, such as spindles, linear guides, ball screws, cutting tools, etc. All the sensors are linked to three data acquisition (DAQ) systems. Each DAQ system performs data acquisition and storage. The data are used for analyzing the correlation between machine health and the quality of the product selected to be machined. Sensor data are captured and recorded in the database during the process of machining the product, which is, in our case, a carburized steel shaft.

As modern machines are quite robust, it may take months or years before detectable changes in machine operation and performance can be observed. Sensors can register small changes indicating the beginning of degradation in machine components or subsystems. However, it is not guaranteed that such changes will affect the quality of machined products. Therefore, it is difficult to determine the remaining useful life of a whole machine system or subsystem based on one-time measurement without access to historical data. In order to obtain the critical and more relevant machine data, different levels of failure modes, such as tool wear, tailstock misalignment, and spindle unbalance, were simulated. At the same time, changes in sensor data collected during experiments to simulate different levels of machine failure modes, as well as the measurement of part quality, including surface roughness, waviness, dimensions, and out-of-roundness, have been captured and documented in order to search for correlations between the sensor signals, deterioration of machine subsystems, and quality of machined products, as shown in Figure 3. To date, a total of 190 GB of data have been collected during the machining of 45 shafts under the different simulated failure modes. Work is ongoing to analyze these data and to generate a model for machine failure prediction.

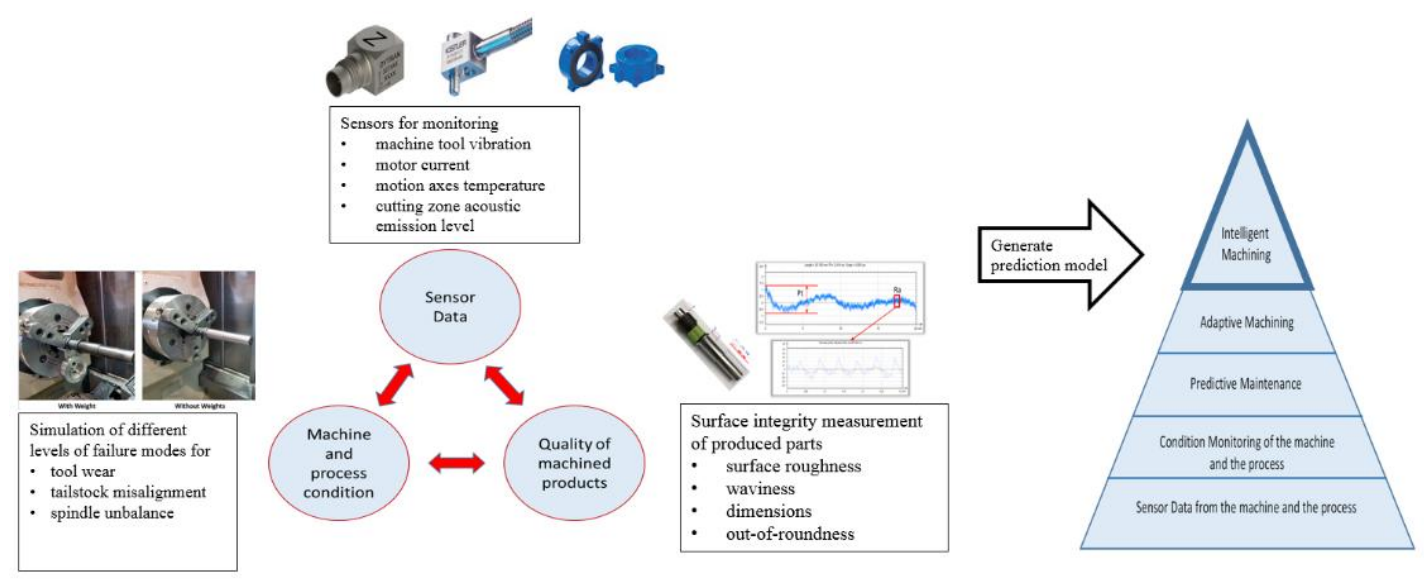

Figure 3. Correlation between machine and process condition, sensor data, and quality of machined products.

The purpose of developing intelligent machining capability is to be able to monitor machine condition and part quality, to predict the need for maintenance, and to avoid costly failure and the need for part rework. In the meanwhile, data captured will also form part of a product's "digital life-story", which will be of great use for supporting product end-of-life decision making and analyzing the cause of product failure. Additionally, the ability to retrofit older machines with smart sensors and the data acquisition system to achieve intelligent machining is of paramount importance, as not all companies can afford to purchase new modern machinery to enjoy the benefits of the sensor system. The capability of retrofitting an existing machine with an advanced sensor system has been developed and demonstrated through this current research work. 


\section{Conclusions}

In this paper, some of the opportunities that Industry 4.0 bring to the remanufacturing industry are discussed and presented from the perspectives of "Smart Life Cycle Data", "Smart Factory", and "Smart Service". It is observed that increased digitalization across the supply chain and enhanced cyber-physical intelligence within the factory have effectively addressed several major concerns that remanufacturers encounter. This will potentially reduce the cost of transformation in remanufacturing.

A smart remanufacturing cell, which provides tailor-made repair operations based on incoming core conditions, is described in the case study section to showcase technology enablers from Industry 4.0 supporting the smart remanufacturing process. Smart sensors which are embedded into the CNC machine enable the real-time monitoring of machine health and call for maintenance or component remanufacturing in a preventative manner. Future work could also look at the economic analysis to justify the viability and profitability of utilizing innovative technologies for a smart remanufacturing system.

Author Contributions: Conceptualization, S.Y., A.R.M.R. and J.K.; Methodology, S.Y.; Supervision, H.P.; Writing-original draft, S.Y., A.R.M.R. and J.K.; Writing-review \& editing, S.Y., A.R.M.R., J.K. and H.P.

Funding: This research received no external funding.

Conflicts of Interest: The authors declare no conflict of interest.

\section{References}

1. World Economic Forum. Towards the Circular Economy: Accelerating the Scale-up across Global Supply Chains. 2014. Available online: http:/ / reports.weforum.org/toward-the-circular-economy-acceleratingthe-scale-up-across-global-supply-chains / (accessed on 28 June 2018).

2. Circular Economy, Ellen Macarthur Foundation. 2018. Available online: https://www. ellenmacarthurfoundation.org/circular-economy (accessed on 28 June 2018).

3. Parker, D.; Riley, K.; Robinson, S.; Symington, H.; Tewson, J.; Jansson, K.; Ramkumar, S.; Peck, D. Remanufacturing Market Study. 2015. Available online: http:/ /www.remanufacturing.eu/assets/pdfs/ remanufacturing-market-study.pdf (accessed on 28 June 2018).

4. APSRG \& APMG, Triple Win-The Social, Economic and Environmental Case for Remanufacturing. 2014. Available online: http://www.policyconnect.org.uk/apsrg/sites/site_apsrg/files/triple_win_-the_social_ economic_and_environmental_case_for_remanufacturing.pdf (accessed on 28 June 2018).

5. Kurilova-Palisaitiene, J.; Lindkvist, L.; Sundin, E. Towards facilitating circular product life-cycle information flow via remanufacturing. Procedia CIRP 2015, 29, 780-785. [CrossRef]

6. Statham, S. Remanufacturing Towards a More Sustainable Future. In Electronics-Enabled Products Knowledge-Transfer Network; Loughborough University: Loughboroug, UK, 2006.

7. PwC. Industry 4.0: Building the Digital Enterprise. 2015. Available online: https://www.pwc.com/gx/ en/industries/industries-4.0/landing-page/industry-4.0-building-your-digital-enterprise-april-2016.pdf (accessed on 28 June 2018).

8. Koch, V.; Kuge, S.; Geissbauer, R.; Schrauf, S. Industry 4.0: Opportunities and Challenges of the Industrial Internet. 2015. Available online: https:/ / www.strategyand.pwc.com/media/file/Industry4.0.pdf (accessed on 28 June 2018).

9. Nasr, N.; Thurston, M. Remanufacturing: A key enabler to sustainable product systems. In Proceedings of the 13th CIRP International Conference on Life-Cycle Engineering, Leuven, Belgium, 31 May-2 June 2006; pp. 15-18.

10. Amezquita, T.; Hammond, R.; Salazar, M.; Bras, B. Characterizing the remanufacturability of engineering systems. In Proceedings of the ASME Advances in Design Automation Conference, Boston, MA, USA, 17-20 September 1995; pp. 271-278.

11. Ijomah, W.; McMahon, C.; Hammond, G.; Newman, S. Development of robust design-for-remanufacturing guidelines to further the aims of sustainable development. Int. J. Prod. Res. 2007, 45, 4513-4536. [CrossRef]

12. Sundin, E. Product and Process Design for Successful Remanufacturing. Ph.D. Thesis, Linköping University, Linköping, Sweden, 2004. 
13. Anderson, D.M. Design for Manufacturability: How to Use Concurrent Engineering to Rapidly Develop Low-Cost, High-Quality Products for Lean Production; CRC Press: Boca Raton, FL, USA, 2014.

14. Calleja-Ochoa, A.; Gonzalez-Barrio, H.; Polvorosa-Teijeiro, R.; Ortega-Rodriguez, N.; Lopez-de-LacalleMarcaide, L.N. Multitasking machines: evolution, resources, processes and scheduling. DYNA 2017, 92, 637-642.

15. Urbikain, G.; Perez, J.M.; López de Lacalle, L.N.; Andueza, A. Combination of friction drilling and form tapping processes on dissimilar materials for making nutless joints. Proc. Inst. Mech. Eng. Part B 2018, 232, 1007-1020. [CrossRef]

16. Pereira, O.; Martín-Alfonso, J.E.; Rodríguez, A.; Calleja, A.; Fernández-Valdivielso, A.; de Lacalle, L.L. Sustainability analysis of lubricant oils for minimum quantity lubrication based on their tribo-rheological performance. J. Clean. Prod. 2017, 164, 1419-1429. [CrossRef]

17. Guidat, T.; Barquet, A.P.; Widera, H.; Rozenfeld, H.; Seliger, G. Guidelines for the definition of innovative industrial product-service systems (PSS) business models for remanufacturing. Procedia CIRP 2014, 16, 193-198. [CrossRef]

18. Rese, M.; Everhartz, J. Condition Monitoring of Industrial Product Service Systems-Helpful selling argument or potential marketing pitfall? In The Philosopher's Stone for Sustainability; Springer: Berlin/Heidelberg, Germany, 2013; pp. 493-497.

(C) 2018 by the authors. Licensee MDPI, Basel, Switzerland. This article is an open access article distributed under the terms and conditions of the Creative Commons Attribution (CC BY) license (http:/ / creativecommons.org/licenses/by/4.0/). 